\title{
Corticosteroids treatment in severe patients with COVID-19: a propensity score matching study
}

\author{
Qian Chen ${ }^{a \#}$, Yang Song ${ }^{\mathrm{b} \#,}$, Lu Wang ${ }^{c}$, Yipeng Zhang ${ }^{\mathrm{b}}$, Lu Han ${ }^{\mathrm{b}}$, Jingru Liü ${ }^{\mathrm{b}}$, Mengyu Yang ${ }^{\mathrm{b}}$, Jingdong Ma ${ }^{\mathrm{b}}$ \\ and Tao Wang ${ }^{c}$
}

aDepartment of Pharmacy, Tongji Hospital, Tongji Medical College, Huazhong University of Science and Technology, Wuhan, China; ${ }^{\circ}$ School of
Medicine and Health Management, Tongji Medical College of Huazhong University of Science and Technology, Wuhan, China; 'Department of
Respiratory and Critical Care Medicine, Tongji Hospital, Tongji Medical College, Huazhong University of Science and Technology, Wuhan, China

\section{ABSTRACT}

Objectives: To explore the efficacy of corticosteroid treatment in patients with severe COVID-19 pneumonia and the association between corticosteroid use and patient mortality.

Methods: A retrospective investigation was made on the medical records of the patients with severe and critical patients with COVID-19 pneumonia from January to February 2020. First, the patients who received corticosteroid treatment were compared with patients without given corticosteroid treatment. Then, a propensity score matching method was used to control confounding factors. Cox survival regression analysis was used to evaluate the effect of corticosteroid therapy on the mortality of severe and critical patients with COVID-19.

Results: A total of 371 severe and critical patients were included in our analyses. Two hundred and enine patients were treated with corticosteroid therapy. Most of them were treated with methylprednisolone (197[94.3\%]). The median corticosteroid therapy was applied 3 (IQR 2-6) days after admission, 13 (IQR 10-17) days after symptoms appeared. Temperature on admission (OR $=1.255,[95 \% \mathrm{Cl} 1.021-$ 1.547], $\mathrm{p}=0.032)$, ventilation $(\mathrm{OR}=1.926$, $[95 \% \mathrm{Cl} 1.148-3.269], \mathrm{p}=0.014)$ and ICU admission $(\mathrm{OR}=3.713,[95 \% \mathrm{Cl} 1.776-8.277], \mathrm{p}<0.001)$ were significantly associated with corticosteroids use. After PS matching, the cox regression survival analysis showed that corticosteroid use was significantly associated with a lower mortality rate $(H R=0.592,[95 \% \mathrm{Cl} 0.406-0.862], \mathrm{p}=0.006)$.

Conclusion: Corticosteroid therapy use in severe and critical patients with COVID-19 pneumonia leads to lower mortality but may cause other side effects. Corticosteroid therapy should be used carefully.

\section{ARTICLE HISTORY}

Received 11 October 2020

Accepted 24 November

2020

KEYWORDS

COVID-19 pneumonia; corticosteroid therapy; critical; mortality; severe

\section{Introduction}

The coronavirus disease 2019 (COVID-19) has been considered as an urgent public health crisis worldwide with the rapidly increasing number of confirmed cases and death tolls, since the outbreak in December 2019. It is reported that more than 46 million have contracted the disease, around 1.2 million died, in 190 countries or areas up to 2 November 2020 [1]. The outbreaks lead to a huge demand for hospital beds and impose great challenges for physicians as well. However, the clinical courses and predictors for the outcome of the patients remain to be fully investigated. Currently, while no specific antiviral or immunomodulatory treatment for COVID-19 has proven effective, therapies recommended for patients with COVID-19 are largely aligned with that of other viral pneumonia, mostly consisting of a set of supportive care strategies [2].

Data from several clinical observational investigations show a significant fraction of the hospitalization patients with COVID-19 received corticosteroid treatment as supportive care. The proportion of patients received corticosteroid treatment varied from $18.6 \%$ to $51.0 \%$ [3-7] depending on the settings and severity of illness. However, the role of corticosteroids in COVID-19 patients is controversial. While the guidance for critical care management from the World Health Organization advocates against their use, there are expert consensus and guidelines incorporate corticosteroids in the clinical management of COVID-19 in severe conditions [8]. For instance, A recent meta-analysis shows that corticosteroid therapy leads to lower 28-day all-cause mortality [9]. Chinese experts consensus recommend short-term therapy with low-to-moderate dose corticosteroids in COVID-19 patients with ARDS [10,11]. Waleed Alhazzani et al. suggest using systemic corticosteroids in mechanically ventilated adults with COVID-19 and ARDS [12]. The debate on the use of corticosteroids in patients with COVID-19 indicates the knowledge gap in understanding the benefits and associated adverse effects of these clinical interventions [13].

The current knowledge base on corticosteroid treatment in viral pneumonia is largely built upon previous experience

CONTACT : Tao Wang wt7636@126.com Department of Respiratory and Critical Care Medicine, Tongji Hospital, Tongji Medical College, Huazhong University of Science and Technology, 1095 Jiefang Avenue, Wuhan 430030, People's Republic of China; Jingdong Ma jdma@hust.edu.cn $\mathbf{E}$ School of Medicine and Health Management, Tongji Medical College of Huazhong University of Science and Technology, 1095 Jiefang Avenue, Wuhan 430030, People's Republic of China "Contributed equally to this manuscript as co-first authors 
with severe patients infected by SARS, MERS, and H1N1, and the treatment effect on clinical outcomes is inconclusive. A retrospective study revealed that proper use of corticosteroids in critical SARS patients was associated with a lowered mortality and shorter length of hospital stay without significant secondary lower respiratory infection and other complications [14]. In an observational study in patients with MERS, corticosteroid therapy did not result in a difference in mortality after adjustment for time-varying confounders but led to delayed MERS coronavirus RNA clearance [15]. Inconsistent results also exist in the studies of influenza viral pneumonia $[16,17]$. To date, few studies have investigated the impact of corticosteroid treatment in patients with COVID-19. Experience from Korea suggests that low-dose steroid oral tablets/inhalers at the earlier stage of COVID-19 and highdose steroid treatment according to the severity of the disease can play important roles in decreasing fatality and pulmonary fibrosis [18]. Zheng et al. analyzed 55 medical records of COVID-19 patients and concluded that early and shortterm use of low-dose methylprednisolone was beneficial and did not delay SARS-CoV-2 RNA clearance and influence IgG antibody production [19]. An observational study in 31 patients reported there were no associations between corticosteroid therapy and outcomes in patients without acute respiratory distress syndrome [6]. Recently a systematic review reports that mortality from corticosteroid use in COVID-19 patients with ARDS seems lower than who did not use [20], and ARDS is an important factor leading to serious consequences and death. However, these findings may not be reliable due to the very small sample size and weakness in study design.

Coping with the pandemic of COVID-19 is extremely challenging for clinicians. Those who considering corticosteroids for severe patients with COVID-19 must balance the potential reduction in mortality with the potential downsides. Understanding the evidence for harm or benefit from corticosteroids in COVID-19 is of immediate clinical importance. However, data from studies on corticosteroid treatment in COVID 19 of sufficient sample size and carefully controlling potential confounding factors and biases are lacking. Therefore, the objective of this study is to identify the factors associated with corticosteroid use and its impact on outcomes in severe patients with COVID-19 accounting for potential selection bias by propensity score matching analysis.

\section{Materials and methods}

\subsection{Study settings and participants}

This is a retrospective observational study. We collected all the 718 cases confirmed with COVID 19 admitted to Zhongfa Xincheng Branch of Tongji Hospital affiliated to Tongji Medical College, Huazhong University of Science and Technology, from Jan 28 to 29 February 2020. Zhongfa Xincheng Branch of Tongji Hospital was temporarily designated to offer 800 beds in refitted isolation wards for severe and critical patients with COVID 19 by the local government during the outbreak. All tests, procedures, therapies were ordered by the attending physician.

The study was approved by the Ethics Committee of Tongji Hospital (IRB: TJ-IRB20200353). Patient identities were protected via anonymization, and the requirement for informed consent was waived due to the observational nature of the study.

\subsection{Data Collection}

The inclusion criteria were patients with a definite diagnosis of Covid-19 based on the World Health Organization interim guidance; patients with a definite outcome (dead or discharged). The exclusion criteria were patients taking chronic corticosteroid therapy for preexisting health conditions; patients receiving corticosteroid therapy as rescue therapy (i.e. due to shock).

Data were collected from the electronic medical records using the standardized International Severe Acute Respiratory and emerging Infection Consortium (ISARIC) case report forms [21]. The extracted variables included patients' demographic characteristics, co-morbidities existing prior to the admission, time of illness onset and hospital admission, signs and symptoms on admission, radiology findings, laboratory test results, medications and supportive care, complications during the hospital stay, and clinical outcomes. Data on the type, maximum daily dose, and duration of corticosteroids were collected.

Four internists (Yipeng Zhang, Lu Han, Jingru Liu, Mengyu Yang) performed the data extraction and data entry independently. The differences between them were detected in the database via an $\mathrm{R}$ code and finally adjudicated by Tao Wang.

\subsection{Study definitions}

Coronavirus disease 2019 (COVID-19) was defined in accordance with the World Health Organization interim guidance. Nasal and pharyngeal swab specimens were collected for detecting SARS-COV-2, which were tested via rt-PCR according to the World Health Organization interim guidance on laboratory testing for COVID-19 in suspected human cases.

Severe and critical patients with COVID-19 were defined following Chinese interim guidelines for diagnosis and treatment for COVID-19 patients (version 7.0). Severe patients were those who had at least one of the clinical features including respiratory rate exceeding 30 breaths/minute at rest, oxygen saturation $\leq 93 \%$ without oxygen support, arterial oxygen partial pressure/fractional inspired oxygen (PaO2/FiO2) $\leq 300 \mathrm{mmHg}$ at rest, the total lesions on chest CT rapid progress $\geq 50 \%$ within $24-48$ hours. Critical patients were those who had at least one of the clinical features including respiratory failure requiring mechanical ventilation, presence of shock, complicating with other organ failures.

Corticosteroid treatment was defined as the use of systemic corticosteroids ordered by the attending physician. All the dosages were converted into methylprednisolone equivalent doses (hydrocortisone 5:1, dexamethasone 1:5, prednisolone 5:4). High-dose corticosteroid therapy was defined as the 
highest daily dose of $\geq 80 \mathrm{mg}$ of methylprednisolone equivalent. Low to moderate dose corticosteroid therapy was defined as the highest daily dose of $<80 \mathrm{mg}$ of methylprednisolone equivalent. Early use of corticosteroid was defined as corticosteroid therapy within 7 days after hospital admission.

\subsection{Endpoints}

The primary objective of this study is to determine whether corticosteroid use was associated with hospital mortality. Additionally, the primary outcome was examined in the following subgroups according to clinical features on hospital admission: (1) severity of illness (severe group vs critical group based on the previous definition); (2) requiring mechanical ventilation on hospital admission (yes vs no); (3) Asthma/ chronic pulmonary disease (yes or not); and (4) inflammatory response to C-reactive protein (CRP $<25$ vs. $\geq 25 \mathrm{mg} / \mathrm{dL}$ ).

The secondary objective of this study is to investigate risk factors associated with corticosteroid use in severe patients with COVID-19. Hospital length of stay, ICU length of stay, mechanical ventilation days, SARS-COV-2 RNA clearance, and presence of complications were also examined in survivors between groups receiving and not receiving corticosteroid treatment.

\subsection{Statistical analysis}

Continuous variables were presented as means with standard deviation (SD) or medians and interquartile range $25-75 \%$ (IQR) and categorical variables as counts (percentage). For demographic variables, baseline clinical characteristics, cointerventions, and outcome variables, differences between patients received corticosteroid treatment during hospitalization and those who did not receive any corticosteroid treatment were compared via Student t-test or Mann-Whitney $U$ test for continuous variables and the chi-square test or Fisher exact test for categorical variables as appropriate.

Multiple logistic regression model was used to examine the association between baseline.

Variables on hospital admission and corticosteroid use. A set of priority-decided variables of clinical interest $[7,15,22]$ and all significant variables at the univariable level $(P<0.2)$ were included in the multivariate model. The probabilities for the entry and removal of variables in a stepwise forward manner were 0.05 and 0.10 , respectively. Model integrity was tested by standard diagnostic statistics and plots; the goodness of fit for each model was assessed with the HosmerLemeshow test. Cox proportional hazards models were used to examine outcomes as a time to event (i.e., death, hospital discharge, SARS-COV-2 RNA clearance, presence of complications), with corticosteroid therapy as a time-varying covariate and adjusting for the same above-mentioned baseline covariates.

To minimize the effect of a corticosteroid treatment selection bias and to control for potential confounding factors, a propensity score matching procedure was performed. The treatment group (patients receive corticosteroid treatment) and control group (those who did not receive corticosteroid treatment) were fully matched with weights based on a full- matching propensity score analysis. The propensity scores were determined using multivariate logistic regression models, which included a set of prespecified covariates without considering the outcomes. C-statistics were used to assess the model's discrimination. The treatment and control pairs were matched via the nearest neighbor matching approach. To ensure balanced matches, a caliper, maximum acceptable difference between two groups, was set as 0.2 (i.e., 0.2 $X$ standard deviation of the logit of the propensity scores) resulting in a relatively narrow difference between matched patients. In addition, the paired t-test or Wilcoxon signed-rank test for continuous as appropriate, and the McNemar test for categorical variables was conducted, respectively, to assess the covariate balance between the treatment and control groups.

After the matching, a Kaplan-Meier survival plot was generated to track hospital mortality over time for the treatment and control group. And the Cox regression model mentioned above was performed again to examine the impact of corticosteroid treatment on patient outcomes after balancing the potential confounding factors and controlling for selection bias.

Finally, a series of subgroup analyses were conducted. Because a corticosteroid therapy effect may be dosedependent, may vary according to the time of initiation, and may vary according to the length of use. We performed all previous models on the following stratified groups: patients who received different doses of corticosteroid therapy, patients who had different corticosteroid initiation time, and patients who had different lengths of corticosteroid therapy. We also compared days for virus clearance between different subgroups and survival time of the death group.

All tests were two-sided, and $p$-values $<0.05$ were considered statistically significant. Results from logistic or Cox regression analysis were reported as odds ratios (OR) or hazard ratios (HR) with 95\% confidence intervals (Cls) as appropriate. Data analysis was performed with the $\mathrm{R}$ software package (cran. r-project.org).

\section{Results}

During the observation period, a total of 762 patients were admitted for COVID-19 infection. After data cleaning, 371 samples met our inclusion criteria of severe or critical patients and were included in this study. Data cleaning path is shown in Figure 1.

\subsection{The comparison between subjects with and without corticosteroid treatment}

Clinical characteristics between the corticosteroid group and the non-corticosteroid group are given in Table 1. According to the initial condition, laboratory tests, and clinical records, patients with corticosteroid treatment are more severe than patients without corticosteroid treatment. Compared with the noncorticosteroid group, the corticosteroid group had higher LDH $(P<0.001), C R P(P<0.001)$, and $D$-dimer $(P<0.001)$, and lower platelets $(P=0.025)$. Antiviral $(P=0.029)$, interferon $(P<0.001)$, antibiotics $(P<0.001)$, and inotropes/vasopressors use 


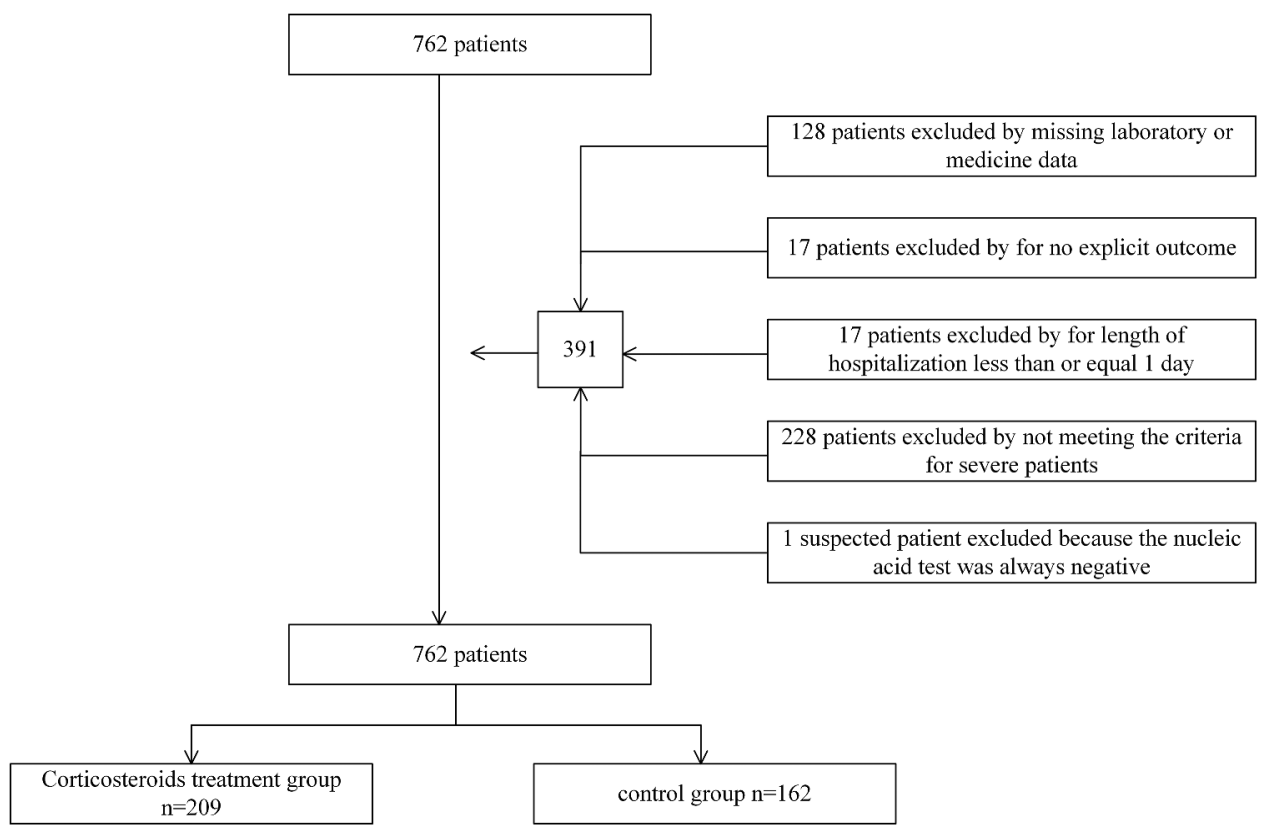

Figure 1. Data cleaning path for all excluded and included patients.

Table 1. Clinical characteristics of patients with COVID-19 in the study.

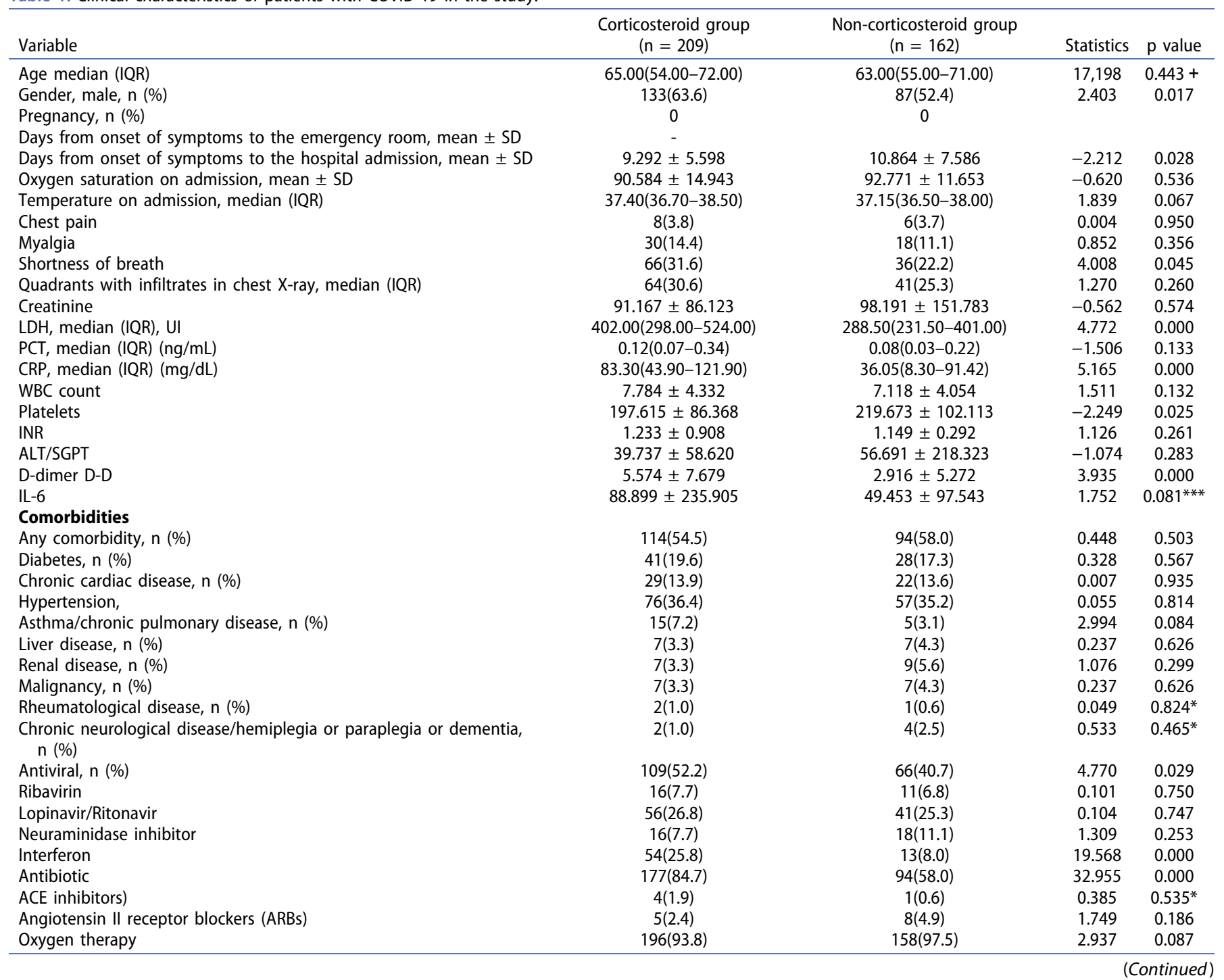


Table 1. (Continued).

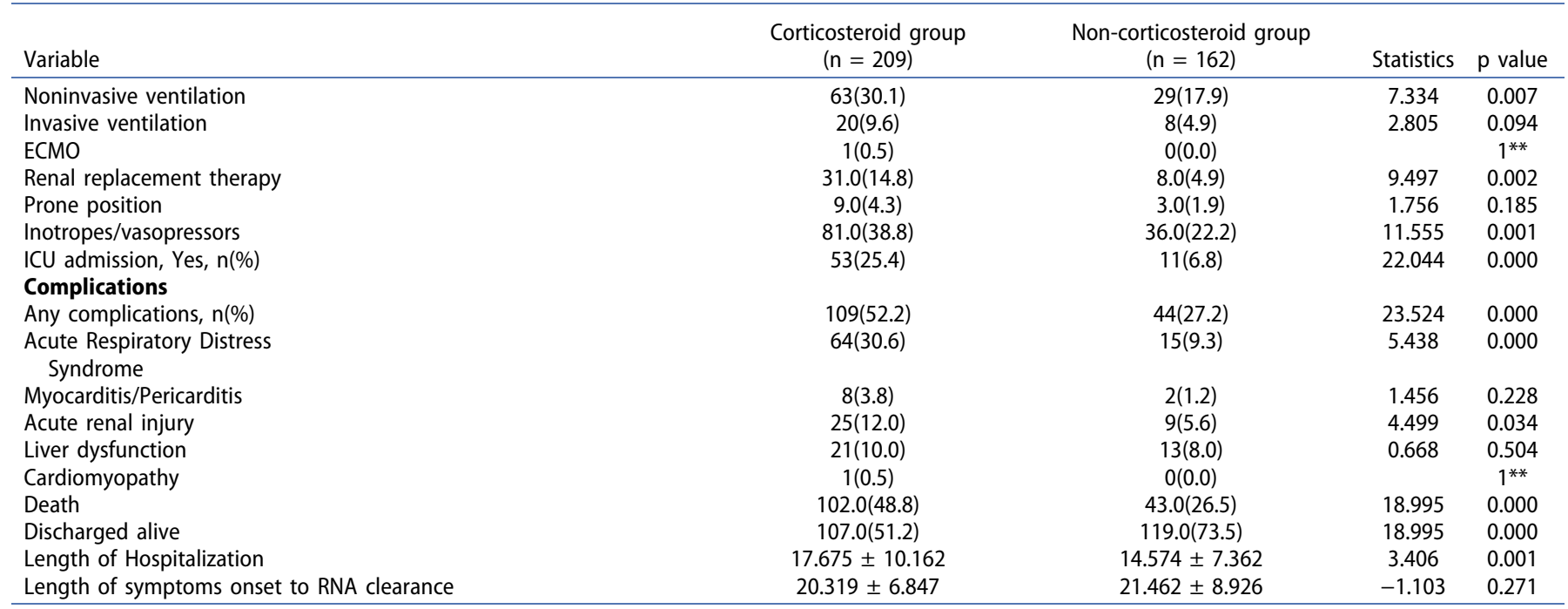

+ Mann-Whitney U test.

${ }^{*}$ Chi-squared test with continuity correction.

**Fishers' exact test.

***Not all patients took laboratory test of IL-6, 289 patients were included Student-t test.

$(P=0.001)$ are more frequent than the non-corticosteroid group. Renal replacement $(P=0.002)$ and noninvasive ventilation therapy use $(P=0.007)$ more than non-corticosteroid group. Complications $(P<0.001)$, ICU admission $(P<0.001)$, length of hospitalization $(P=0.001)$, and overall death rate $(P<0.001)$ are larger than non-corticosteroid group. All of this suggests that patients with corticosteroid therapy are more critical.

Two hundred and nine patients received corticosteroid therapy during hospitalization. Of these, 197 (94.3\%) patients were treated with Methylprednisolone, 39 (18.7\%) prednisone, 6 (2.8\%) methylprednisolone tablets, and 1(0.5\%) dexamethasone. ${ }^{1}$ After admission, the median (IQR) corticosteroid therapy was applied 3 (2-6) days later, 13 (10-17) days after symptoms appeared. Among all patients with corticosteroid treatment, the median duration of corticosteroid therapy is 7(4-12) days and 5(3-8.75) for the survivor group and 10 (7-14) for the death group. Details are shown in Table 2.

\subsection{Factors for corticosteroid use in patients with COVID-19}

To clarify which factors influencing corticosteroid treatment use, we selected indicators for patients before corticosteroid therapy with discretion to build a stepwise logistic regression model. Age, critical, the temperature on admission, ventilation, and ICU admission were included finally. Temperature on admission ( $O R=1.255,[95 \% \mathrm{Cl} 1.021-1.547], p=0.032)$, ventilation $(\mathrm{OR}=1.926,[95 \% \mathrm{Cl} 1.148-3.269], \mathrm{p}=0.014)$ and ICU admission $(\mathrm{OR}=3.713,[95 \% \mathrm{Cl} 1.776-8.277], \mathrm{p}<0.001)$ are significant. Detail results are shown in Table 3.
Table 2. Administration of corticosteroids in patients with COVID 19.

Drug administered, $\mathrm{n}(\%)^{*}$

Prednisone 39(10.5)

Methylprednisolone 197(53.1)

Methylprednisolone tablet

6(1.6)

Dexamethasone

$1(0.3)$

Initial dosage (equivalent Methylprednisolone), $\mathrm{mg} / \mathrm{d}$

Mean \pm SD

Median (IQR)

$49.456 \pm 24.361$

Maximum dosage (equivalent

Methylprednisolone), $\mathrm{mg} / \mathrm{d}$

Mean \pm SD

Median (IQR)

Time to initiation from hospitalization, $d$

Mean \pm SD

Median (IQR)

Time to initiation from symptoms onset, $d$

Mean \pm SD

Median (IQR)

Duration of therapy (d)(all patients)

Mean \pm SD

Median (IQR)

Duration of therapy (d) (Survivors)

Mean \pm SD

Median (IQR)

Duration of therapy (d) (Non-Survivors)

Mean \pm SD

Median (IQR)

$\mathrm{PaO2} / \mathrm{FIO} 2$ before corticosteroid initiation

Mean \pm SD

Median (IQR) $40.00(40.00-80.00)$

$63.785 \pm 41.838$

$40.00(40.00-80.00)$

$4.833 \pm 4.818$

$3.00(2.00-6.00)$

$14.124 \pm 6.420$ $13.00(10.00-17.00)$

$9.144 \pm 7.501$

$7.00(4.00-12.00)$ $(\mathrm{n}=107)$

$11.533 \pm 7.745$

$5.000(3.000-8.750)$

$(\mathrm{n}=102)$

$6.637 \pm 6.365$ 10.000

(7.000-14.000)

$(\mathrm{n}=34)$

$379.267 \pm 212.574$ 323.81

(247.62-440.48)

*Some patients changed corticosteroid type during treatment process. For rigors' sake, here is the statistics of patients who have used this kind of corticosteroid drug. 
Table 3. Multivariate analysis for factors associated with corticosteroid therapy (stepwise).

\begin{tabular}{lccr}
\hline Variable & OR & $95 \% \mathrm{Cl}$ & $\mathrm{p}$-value \\
\hline Age & 0.988 & $0.971-1.004$ & 0.148 \\
Critical & 1.576 & $0.872-2.856$ & 0.132 \\
Temperature on admission & 1.255 & $1.021-1.547$ & 0.032 \\
Ventilation & 1.926 & $1.148-3.269$ & 0.014 \\
ICU admission & 3.713 & $1.776-8.277$ & $<0.001$ \\
\hline
\end{tabular}

\subsection{Mortality analysis}

One hundred and two of 209 (48.8\%) patients with corticosteroid treatment died, which mortality rate is higher than the non-corticosteroid group (43 of $162,26.5 \%$, chi-squared test $p<0.001)$. After controlling the factors that may affect the outcome, there is no significant evidence for corticosteroid therapy affects outcome $(\mathrm{OR}=0.969$, [95\%Cl $0.352-2.606]$, $p=0.950)$.

To minimization selection bias, a PS-full matching model was applied to our dataset and every sample in the non-corticosteroid group and corticosteroid group was given a PS full matching weights. Age, gender, the temperature on admission, days from symptom onset to admission, critical on admission, $\mathrm{LDH} \geq 225 \mathrm{U} / \mathrm{L}$, $\mathrm{CRP} \geq 1 \mathrm{mg} / \mathrm{L}$, D-Dimer $\geq 0.5 \mathrm{ug} / \mathrm{mL}$, any complications, chronic heart disease, chronic liver disease, chronic renal disease, chronic neurological disease, hypertension, hyperlipidemia, renal replacement therapy, ICU on admission were the independent variables included in the logistic regression analysis of the PS-full matching model. Summaries of balance for matched data and unmatched data are shown in Table 4.

After PS-full matching, we performed a logistic regression model and a Cox regression model to determine the impact of corticosteroid use in severe patients with COVID-19. With PSfull matching, the logistic regression model showed that corticosteroid use is linked to a lower mortality rate $(O R=0.308$, [95\% Cl 0.112-0.771], $\mathrm{p}=0.016)$. Our cox regression model (Table 5) and adjusted cox regression survival plot (Figure 3) confirm that corticosteroid use was significantly associated with a lower mortality rate $(\mathrm{HR}=0.592,[95 \% \mathrm{Cl} 0.406-0.862]$, $p=0.006)$.

To make the results more robust, we performed several subgroup analyses. First, we separated the corticosteroid group by a maximum dosage of corticosteroid use, days from admission to corticosteroid use, and total days of corticosteroid use. The subgroup logistic results showed that longer the duration corticosteroid use $(\mathrm{OR}=37.032,[95 \% \mathrm{Cl}$ 2.042-3461.331], $p=0.040$ ) links to higher survival rate, while the maximum dosage $(\mathrm{OR}=0.969,[95 \% \mathrm{Cl} 1.215-1631.377]$,

Table 4. Comparison of baseline characteristics between treated and untreated subjects in the original sample and in the propensity score-matched sample.

\begin{tabular}{|c|c|c|c|c|c|c|}
\hline \multirow[b]{2}{*}{ Baseline variables } & \multicolumn{3}{|c|}{ Original sample } & \multicolumn{3}{|c|}{ Matched sample } \\
\hline & $\begin{array}{l}\text { Treated group } \\
\qquad(n=209)\end{array}$ & $\begin{array}{l}\text { Control group } \\
(n=162)\end{array}$ & Mean difference & $\begin{array}{l}\text { Treated group } \\
\qquad(\mathrm{n}=209)\end{array}$ & $\begin{array}{l}\text { Control group } \\
(n=162)\end{array}$ & Mean difference \\
\hline $\begin{array}{l}\text { Global distance } \\
\text { Demographic data }\end{array}$ & 0.6263 & 0.4821 & 0.1441 & 0.6263 & 0.6258 & 0.0005 \\
\hline Age & 62.5502 & 62.4259 & 0.1243 & 62.5502 & 62.4707 & 0.0796 \\
\hline Temperature & 37.5694 & 37.3642 & 0.2052 & 37.5694 & 37.5960 & -0.0267 \\
\hline Female & 0.6364 & 0.5123 & 0.1240 & 0.6364 & 0.6295 & 0.0068 \\
\hline Male & 0.3636 & 0.4877 & -0.1240 & 0.3636 & 0.3705 & -0.0068 \\
\hline \multicolumn{7}{|l|}{ Severity of illness } \\
\hline Serious & 0.4067 & 0.2037 & 0.2030 & 0.4067 & 0.3322 & 0.0745 \\
\hline Critical & 0.5933 & 0.7963 & -0.2030 & 0.5933 & 0.6678 & -0.0745 \\
\hline \multicolumn{7}{|l|}{ Service Utilization } \\
\hline Days from onset of symptoms to the hospital admission & 9.2919 & 10.8642 & -1.5723 & 9.2919 & 9.0814 & 0.2104 \\
\hline ICU admission & 0.2584 & 0.0741 & 0.1843 & 0.2584 & 0.2416 & 0.0167 \\
\hline \multicolumn{7}{|l|}{ Laboratory } \\
\hline $\mathrm{LDH}$ & 450.7368 & 343.6049 & 107.1319 & 450.7368 & 423.5441 & 27.1927 \\
\hline CRP & 92.4766 & 58.1926 & 34.2840 & 92.4766 & 74.1692 & 18.3073 \\
\hline ALT & 39.7368 & 56.6914 & -16.9545 & 39.7368 & 72.4182 & -32.6814 \\
\hline D-Dimer & 5.5530 & 3.0003 & 2.5526 & 0.1800 & 2.4204 & 18.0200 \\
\hline \multicolumn{7}{|l|}{ Comorbidities } \\
\hline Any comorbidity & 0.5598 & 0.6111 & -0.0513 & 0.5598 & 0.5921 & -0.0323 \\
\hline Diabetes & 0.2010 & 0.1790 & 0.0219 & 0.2010 & 0.2498 & -0.0489 \\
\hline Chronic cardiac disease & 0.1435 & 0.1481 & -0.0046 & 0.1435 & 0.1277 & 0.0159 \\
\hline Hypertension & 0.3732 & 0.3580 & 0.0152 & 0.3732 & 0.3408 & 0.0324 \\
\hline Asthma/chronic pulmonary disease & 0.0718 & 0.0432 & 0.0286 & 0.0718 & 0.0399 & 0.0319 \\
\hline Liver disease & 0.0335 & 0.0494 & -0.0159 & 0.0335 & 0.0386 & -0.0051 \\
\hline Renal disease & 0.0383 & 0.0617 & -0.0235 & 0.0383 & 0.0183 & 0.0200 \\
\hline Malignancy & 0.0287 & 0.0432 & -0.0145 & 0.0287 & 0.0407 & -0.0120 \\
\hline neurological disease & 0.0096 & 0.0247 & -0.0151 & 0.0096 & 0.0070 & 0.0026 \\
\hline \multicolumn{7}{|l|}{ Complications } \\
\hline Any complications & 0.5215 & 0.2778 & 0.2438 & 0.5215 & 0.5230 & -0.0015 \\
\hline $\begin{array}{l}\text { Acute Respiratory Distress } \\
\text { Syndrome }\end{array}$ & 0.3062 & 0.0926 & 0.2136 & 0.3062 & 0.2241 & 0.0821 \\
\hline Myocarditis/Pericarditis & 0.0431 & 0.0123 & 0.0307 & 0.0431 & 0.0215 & 0.0215 \\
\hline Acute renal injury & 0.1196 & 0.0556 & 0.0641 & 0.1196 & 0.1313 & -0.0117 \\
\hline Liver dysfunction & 0.1005 & 0.0802 & 0.0202 & 0.1005 & 0.1581 & -0.0576 \\
\hline
\end{tabular}


Table 5. Cox survival regression result after PS-full matching.

\begin{tabular}{lccc}
\hline Variable & $\mathrm{HR}$ & $95 \% \mathrm{Cl}$ & $\mathrm{p}$-value \\
\hline Corticosteroid group & 0.592 & $0.406-0.862$ & 0.006 \\
Age & 1.034 & $1.014-1.054$ & 0.000 \\
Temperature on admission & 1.018 & $0.858-1.208$ & 0.835 \\
Gender male & 1.497 & $0.961-2.329$ & 0.073 \\
Critical & 1.370 & $0.680-2.760$ & 0.378 \\
Ventilation & 2.632 & $1.879-3.685$ & 0.000 \\
$\mathrm{LDH} \geq 225 \mathrm{U} / \mathrm{L}$ & 1.979 & $0.624-6.266$ & 0.245 \\
$\mathrm{CRP} \geq 1 \mathrm{mg} / \mathrm{L}$ & 0.913 & $0.238-3.498$ & 0.894 \\
$\mathrm{D}-\mathrm{Dimer} \geq 0.5 \mathrm{ug} / \mathrm{mL}$ & 2.135 & $0.926-4.919$ & 0.074 \\
ARDS & 1.564 & $0.909-2.690$ & 0.105 \\
Acute renal failure & 1.003 & $0.586-1.716$ & 0.991 \\
Liver dysfunction & 1.159 & $0.599-2.238$ & 0.661 \\
Renal replacement therapy & 0.774 & $0.496-1.206$ & 0.257 \\
Chronic liver disease & 1.181 & $0.425-3.279$ & 0.749 \\
Chronic renal disease & 1.566 & $0.600-4.084$ & 0.359 \\
Bacterial pneumonia & 0.449 & $0.124-1.613$ & 0.219 \\
Bacteremia & 6.309 & $2.872-13.857$ & 0.000 \\
Hypertension & 1.627 & $1.016-2.603$ & 0.042 \\
Hyperlipidemia & 2.804 & $0.466-16.855$ & 0.259 \\
Chronic neurological disease & 1.766 & $2.344-14.581$ & 0.000 \\
Antivirus & 0.770 & $0.517-1.146$ & 0.198 \\
Antibiotic & 1.349 & $0.620-2.932$ & 0.450 \\
ICU admission & 1.382 & $0.770-2.478$ & 0.278 \\
\hline
\end{tabular}

$p=0.081)$ and early or late start of treatment $(O R=21.539$, [95\%Cl 0.160-6.273], $\mathrm{p}=0.973$ ) are not significant (Figure 2 part1). In the subgroup of patients who survived with a clear timeline for virus clearance, corticosteroid therapy has no significant influence $(\beta=1.148,[95 \% \mathrm{Cl}-32.223-17.662]$, $p=0.131)$ on virus clearance, but long-term use $(\beta=1.738$, [95\% Cl $-0.017-3.493], p=0.052$, weak significance) and lateuse $(\beta=3.729,[95 \% \mathrm{Cl} 0.871-6.587], \mathrm{p}=0.011$ ) may cause more day for virus clearance. As for the death group, corticosteroid therapy earned more survival time $(\beta=3.463,[95 \% \mathrm{Cl}$ $0.591-6.336], p=0.019$ ) for other clinical interventions. Details are shown in Figure 2 part2.

\section{Discussion}

Our results confirm that corticosteroid therapy in severe and critical patients with COVID-19 is associated with a lower mortality rate, which is consistent with several recent researches $[9,23]$. Although it appears that the mortality rate of the corticosteroid group is higher than the control group, we found that corticosteroid therapy reduces the mortality rate after balancing the condition of patients by PS full matching.

Corticosteroids therapy in pneumonia and COVID-19 pneumonia remains a controversy. Corticosteroid has functions of anti-inflammatory, anti-allergic and hypothermic. Cytokine release syndrome (CRS) in COVID-19 often leads to multiorgan dysfunctions and links to the severity of the syndrome and the outcome [24-26]. Therefore, it is suggested that using corticosteroid as adjunctive therapy in COVID-19 treatment. On the other hand, due to its immunosuppression property, potential risks of corticosteroid therapy including secondary infections, long-term complications, and delayed virus clearance prevent doctors from using Corticosteroid therapy [25].

Scientists have ambiguous opinions on corticosteroid therapy that affect COVID-19 pneumonia. A variety of articles claim it benefits or stays neutrality [27-33]; meanwhile, there is also evidence that corticosteroid treatment is associated with high mortality and it is recommended not to use corticosteroid [34-37]. Given that the above studies did not exclude endogenous problems well and COVID-19 is still in the epidemic and based on conflicting perspectives, we aimed at severe and critical patients with COVID-19 pneumonia and balance patient conditions before corticosteroid treatment by PS full matching to perform a more robust result whether corticosteroid works on severe and critical patients with COVID-19.

In our results, corticosteroid treatment was significant benefits to the survival of severe and critical patients with COVID-19 pneumonia after balancing the selection bias, although it seems that the corticosteroid group has a higher mortality rate at first glance (unbalanced). Most retrospective research has ignored the selection bias and did not provide robust results. However, physicians consider whether to use corticosteroids often refers to the severity of illness, which leads to serious endogenous problems. To the best of our knowledge, no study using a similar analysis to examine corticosteroid treatment in severe and

\begin{tabular}{|c|c|c|c|c|c|c|c|}
\hline subgroup & dependent & coef & yes $n$ & no & & $\mathbf{P}$ & $\beta(95 \% \mathrm{Cl})$ \\
\hline \multirow[t]{4}{*}{$\begin{array}{l}\text { discharged } \\
\text { timeline for }\end{array}$} & days for virus clear & & & & & & \\
\hline & & corticosteroid therapy & 10911 & $115 \leftarrow$ & - & 0.132 & $1.147(-32.223 \sim 17.662)$ \\
\hline & & $\begin{array}{c}\text { total days of } \\
\text { corticosteroid use }>7\end{array}$ & & 82 & $\rightarrow-1$ & 0.052 & $1.738(-0.017 \sim 3.493)$ \\
\hline & & $\begin{array}{l}\text { days from admission } \\
\text { to corticosteroid use }>=5\end{array}$ & & 13 & $\longmapsto-1$ & 0.011 & $3.729(0.872 \sim 6.587)$ \\
\hline \multirow[t]{2}{*}{ death } & survival time & & & & & & \\
\hline & & corticosteroid therapy & 10243 & 43 & $\longmapsto-1$ & 0.019 & $3.463(0.591 \sim 6.336)$ \\
\hline
\end{tabular}

Figure 2. (1) Subgroup logistic regression of different usage of corticosteroid affects survival rate. (2) Subgroup linear regression of different usage of corticosteroid affects days for virus clearance or survival time. 


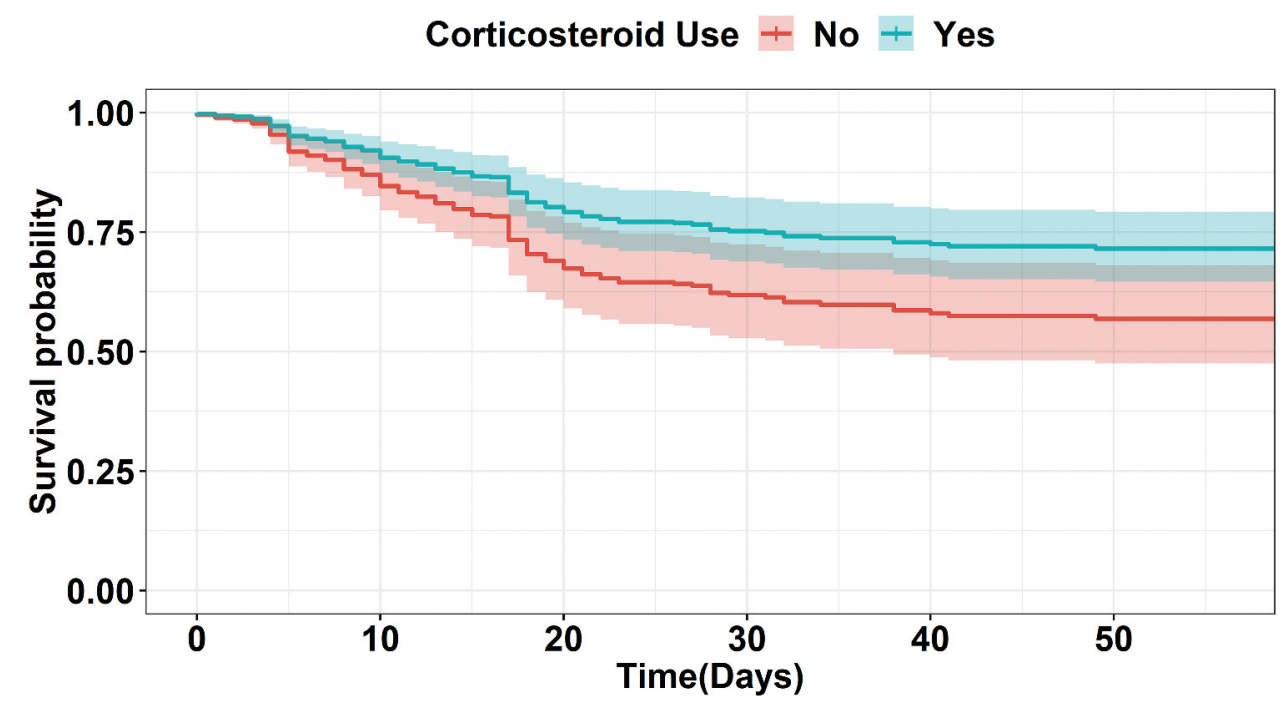

Figure 3. Cox regression survival plot according to corticosteroid treatment use.

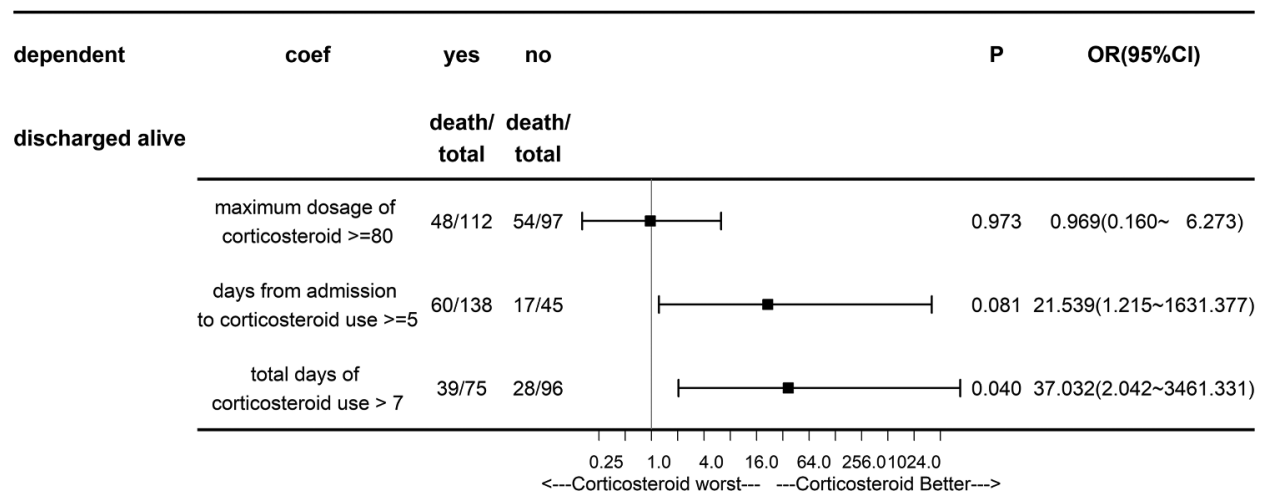

critical patients with COVID-19 pneumonia. Only one study uses 1:1 PS matching on not severe patients with COVID-19 infected. By a group of 70 matched patients, Yuan et al. [38] concluded that corticosteroid might harm lung injury recovery in nonsevere COVID-19 pneumonia patients. As an effective candidate treatment therapy, the corticosteroid is being concerned by researchers recently [39-41]. However, more attention should be paid to severe and critical patients.

In our subgroup analysis, long-term use of corticosteroids is associated with a higher survival rate. The effect of large-dose use and late use on survival rate seems not significant. But in a group of discharged alive with a clear timeline for virus clearance, long-term use (weak significant $p=0.522$ ) and late use extend the duration of viral shedding, which is consistent with most existing studies and reflects the harm of corticosteroid therapy $[25,31,32]$. Even so, in the death group, corticosteroid also prolongs the survival time of the patients, which gives the doctor more buffer to perform clinical interventions.

This study collects a relatively large dataset to balance selection bias and endogenous by PS full matching method, which makes our research more robust. But there still exist limitations. First, data are collected from single-center and our hospital mainly receives patients with severe illness. Our results and conclusions are only applicable to severe and critical patients with COVID-19 pneumonia. Second, this is a retrospective study, some interesting clinical evidence is not collected such as CT source image, SOFA score, etc. Third, in the early stage, due to the lack of nucleic acid detection reagents, some patients did not have a complete virus clearance trace; thus, we lost part of the data in our subgroup analysis. Finally, the common defect of all retrospective studies is that we can't completely control the existence of bias. However, as PS matching analysis can balance the selection bias and endogenous problems, it is the best evidence available for physicians.

\section{Conclusion}

In a group of severe and critical patients with COVID-19 pneumonia, after balancing selection bias and endogeneity, corticosteroid therapy was significantly associated with decreased mortality. Physicians should use it carefully because it also prolongs the duration of virus clearance. 


\section{Funding}

This work was supported by HUST COVID-19 Rapid Response Call 2020kfyXGYJ015 to Tao Wang.

\section{Note}

1. Some patients changed corticosteroid type during the treatment process. For rigors' sake, here are the statistics of patients who have used this type of corticosteroid drug. Which means that these percentages add up to more than 1 due to some patients received more than 1 type of corticosteroids.

\section{Author contributions}

Study design: Tao Wang, Jingdong Ma. Data extraction and data entry: Lu Wang, Yipeng Zhang, Lu Han, Jingru Liu, Mengyu Yang. Data collection: Qian Chen, Yang Song, Lu Wang. Data analysis: Jingdong Ma, Yang Song. Drafting manuscript: Jingdong Ma, Yang Song. Revising manuscript content: Tao Wang, Jingdong Ma, Yang Song. Approving the final version of the manuscript: Tao Wang, Jingdong Ma, Yang Song. All authors read and approved the final manuscript.

\section{Ethics approval and consent to participate}

The study was approved by the Ethics Committee of Tongji Hospital ((IRB: TJ-IRB20200353)). Patient identities were protected via anonymization, and the requirement for informed consent was waived due to the observational nature of the study. Written informed consent for publication was obtained from all participants.

\section{Data availability}

The dataset used and analyzed during the current study are available from the corresponding author on reasonable request.

\section{Acknowledgments}

We thank all medical staffs and patients involved in the study.

\section{Declaration of interest}

The authors have no relevant affiliations or financial involvement with any organization or entity with a financial interest in or financial conflict with the subject matter or materials discussed in the manuscript. This includes employment, consultancies, honoraria, stock ownership or options, expert testimony, grants or patents received or pending, or royalties.

\section{Reviewer disclosures}

Peer reviewers on this manuscript received an honorarium for their review work. Peer reviewers on this manuscript have no other relevant financial or other relationships to disclose.

\section{References}

1. COVID-19 Map. [cited November 2, 2020]. Available from: https:// coronavirus.jhu.edu/map.html

2. Singhal TA. Review of coronavirus disease-2019 (COVID-19). Indian J Pediatr. 2020;87(4):281-286.

3. Guan W, Ni Z, Hu Y, et al. Clinical characteristics of coronavirus disease 2019 in China. N Engl J Med. 2020;382(18):1708-1720.

4. Huang C, Wang $Y$, Li X, et al. Clinical features of patients infected with 2019 novel coronavirus in Wuhan, China. LANCET. 2020;395 (10223):497-506.
5. Mo $P$, Xing $Y$, Xiao $Y$, et al. Clinical characteristics of refractory COVID-19 pneumonia in Wuhan, China. Clin Infect Dis. 2020 Mar 16. DOI:10.1093/cid/ciaa270.

6. Zha L, Li S, Pan L, et al. Corticosteroid treatment of patients with coronavirus disease 2019 (COVID-19). Med J Aust. 2020;212(9):416-420.

7. Zhou F, Yu T, Du R, et al. Clinical course and risk factors for mortality of adult inpatients with COVID-19 in Wuhan, China: a retrospective cohort study. Lancet. 2020;395(10229):1054-1062.

8. Clinical management of severe acute respiratory infection when novel coronavirus (2019-nCoV) infection is suspected: interim guidance, 28 January 2020. Geneva: World Health Organization; 2020.

9. Sterne JAC, Murthy S, Diaz JV, et al. Association between administration of systemic corticosteroids and mortality among critically ill patients with COVID-19: A Meta-analysis. JAMA. 2020;324 (13):1330-1341.

10. Shang L, Zhao J, Hu Y, et al. On the use of corticosteroids for 2019-nCoV pneumonia. Lancet. 2020;395(10225):683-684.

11. Zhao JP, Hu Y, Du RH, et al. Expert consensus on the use of corticosteroid in patients with 2019-nCoV pneumonia. Chin J Tubercul Respirat Dis. 2020;43(3):183-184.

12. Alhazzani W, Moller MH, Arabi YM, et al. Surviving sepsis campaign: guidelines on the management of critically ill adults with coronavirus disease 2019 (COVID-19). Intensive Care Med. 2020;46(5):854-887.

13. Kow CS, Hasan SS. Corticosteroid-related in-hospital hyperglycemia: does it negate mortality benefits in coronavirus disease 2019? Clin Infect Dis. 2020. DOI:10.1093/cid/ciaa1423

14. Chen RC, Tang XP, Tan SY, et al. Treatment of severe acute respiratory syndrome with glucosteroids: the Guangzhou experience. Chest. 2006;129(6):1441-1452.

15. Arabi YM, Mandourah Y, Al-Hameed F, et al. Corticosteroid therapy for critically ill patients with middle east respiratory syndrome. Am J Respir Crit Care Med. 2018;197(6):757-767.

16. Lansbury LE, Rodrigo C, Leonardi-Bee J, et al. Corticosteroids as adjunctive therapy in the treatment of influenza: an updated cochrane systematic review and meta-analysis. Crit Care Med. 2020;48(2):e98-e106.

17. Zhang $Y$, Sun W, Svendsen ER, et al. Do corticosteroids reduce the mortality of influenza A (H1N1) infection? A meta-analysis. Crit Care. 2015;19:46.

18. Song YG, Shin HS. COVID-19, a clinical syndrome manifesting as hypersensitivity pneumonitis. Infect Chemother. 2020;52 (1):110-112.

19. Zheng C, Wang J, Guo H, et al. Risk-adapted treatment strategy For COVID-19 patients. Inter J Infect Dis. 2020;94:74-77.

20. Hasan SS, Capstick T, Ahmed R, et al. Mortality in COVID-19 patients with acute respiratory distress syndrome and corticosteroids use: a systematic review and meta-analysis. Expert Rev Respir Med. 2020;14(11):1149-1163.

21. Clinical Data Collection. The COVID-19 case report forms (CRFs). [cited November 2, 2020]. Available from: https://isaric.tghn.org/ COVID-19-CRF/

22. Cao B, Gao H, Zhou B, et al. Adjuvant corticosteroid treatment in adults with influenza a (H7N9) viral pneumonia. Crit Care Med. 2016;44(6):E318-E328.

23. Angus DC, Derde L, Al-Beidh F, et al. Effect of hydrocortisone on mortality and organ support in patients with severe COVID-19: the REMAP-CAP COVID-19 corticosteroid domain randomized clinical trial. JAMA. 2020;324(13):1317-1329.

24. Conti $P$, Ronconi G, Caraffa A, et al. Induction of pro-inflammatory cytokines (IL-1 and IL-6) and lung inflammation by coronavirus-19 (COVI-19 or SARS-CoV-2): anti-inflammatory strategies. J Biol Regul Homeost Agents. 2020;34(2):327-331.

25. Dai J, Xiong $\mathrm{Y}, \mathrm{Li} \mathrm{H}$, et al. Corticosteroid treatment in severe COVID-19 pneumonia: two cases and literature review. Clin Rheumatol. 2020;39(7):2031-2037.

26. Liu F, Li L, Xu M, et al. Prognostic value of interleukin-6, C-reactive protein, and procalcitonin in patients with COVID-19. J Clin Virol. 2020;127:104370. 
27. Fang X, Mei Q, Yang T, et al. Low-dose corticosteroid therapy does not delay viral clearance in patients with COVID-19. J Infect. 2020;81(1):147-178.

28. Kolilekas L, Loverdos K, Giannakaki S, et al. Can steroids reverse the severe COVID-19 induced 'cytokine storm'? J Med Virol. 2020;92 (11):2866-2869.

29. Maes T, Bracke K, Brusselle GGCOVID-19. COVID-19, asthma, and inhaled corticosteroids: another beneficial effect of inhaled corticosteroids? Am J Respir Crit Care Med. 2020;202(1):8-10.

30. So C, Ro S, Murakami M, et al. High-dose, short-term corticosteroids for ARDS caused by COVID-19: a case series. Respirol Case Rep. 2020;8(6):e00596-e00596.

31. Taboada M, Caruezo V, Naveira A, et al. Corticosteroids and the hyper-inflammatory phase of the COVID-19 disease. J Clin Anesth. 2020;66:109926.

32. Veronese N, Demurtas J, Yang L, et al. Use of corticosteroids in coronavirus disease 2019 pneumonia: a systematic review of the literature. Front Med (Lausanne). 2020;7:170.

33. Ye Z, Wang $Y$, Colunga-Lozano LE, et al. Efficacy and safety of corticosteroids in COVID-19 based on evidence for COVID-19, other coronavirus infections, influenza, community-acquired pneumonia and acute respiratory distress syndrome: a systematic review and meta-analysis. CMAJ = Journal De I'Association Medicale Canadienne. 2020;192(27):E756-E767.

34. Li X, Xu S, Yu M, et al. Risk factors for severity and mortality in adult COVID-19 inpatients in Wuhan. J Allergy Clin Immunol. 2020;146 (1):110-118.
35. Russell B, Moss C, Rigg A, et al. COVID-19 and treatment with NSAIDs and corticosteroids: should we be limiting their use in the clinical setting? ECANCERMEDICALSCIENCE. 2020;14:1023.

36. Wang D, Hu B, Hu C, et al. Clinical characteristics of 138 hospitalized patients with 2019 novel coronavirusinfected pneumonia in Wuhan, China. JAMA. 2020;323(11):1061-1069.

37. Yang Z, Liu J, Zhou Y, et al. The effect of corticosteroid treatment on patients with coronavirus infection: a systematic review and meta-analysis. J Infect. 2020;81(1):e13-e20.

38. Yuan $M, X u X, X i a ~ D$, et al. Effects of corticosteroid treatment for non-severe COVID-19 pneumonia: a propensity score-based analysis. Shock. 2020;54(5):638-643.

39. Matthay MA, Wick KD. Corticosteroids, COVID-19 pneumonia and acute respiratory distress syndrome. J Clin Invest. 2020. DOI:10.1172/JCI143331

40. Saghazadeh A, Rezaei N. Towards treatment planning of COVID-19: rationale and hypothesis for the use of multiple immunosuppressive agents: anti-antibodies, immunoglobulins, and corticosteroids. Int Immunopharmacol. 2020 Jul;84:106560.

41. Singh AK, Majumdar S, Singh R, et al. Role of corticosteroid in the management of COVID-19: A systemic review and a Clinician's perspective. Diabetes Metab Syndr. 2020 Sep;14 (5):971-978. 